\title{
The mitochondrial basis of aging and related diseases
}

\author{
Junxi Mou ${ }^{1, \mathrm{a}}$ \\ ${ }^{1}$ Guanghua Cambridge International School, Shanghai, China
}

\begin{abstract}
Aging is a programmed and spontaneous life course that organisms must undergo, and as an irreversible and relatively conservative process, several theories have tried to explain its causes. Among them, the somatic mutation theory, the free radical theory, the natural cross-linking of biomolecules, the immune theory, the telomere theory, the biological clock theory, and the toxic theory are among the most widely accepted hypotheses. However, no single doctrine can fully explain the aging process. Aging is mainly manifested in the structural and functional aging and decline of organisms, and as organisms continue to age, most organs will experience varying degrees of aging and irreversible damage, although it is directly related to many diseases, aging itself is generally not considered as a disease. Any organism ages slowly over time, with the most easily observable manifestations in external changes such as diminished hair color and sagging skin. Numerous studies have shown that mitochondria are instrumental in mitigating aging as the primary provider of cellular energy, providing energy for cell growth and development as well as being the center of cellular metabolism; therefore, once mitochondria become faulty, a large number of cells will lose function, age, and thus die. In this paper, we will discuss in detail the relationship between mitochondria and aging and how external interventions can promote the function of mitochondria in delaying aging.
\end{abstract}

\section{Introduction}

Mitochondria are ordered and highly specialized organelles present in eukaryotic cells. To accommodate their functional diversity, mitochondria exhibit different morphological features: sometimes threadlike and sometimes splitting into granules. Its main function is to serve as the main site of the intracellular tricarboxylic acid cycle (TCA), oxidative phosphorylation (oxygen consumption) and ATP production. The production of energy may be accompanied by excessive production of reactive oxygen radicals (ROS), leading to disruption of mitochondrial metabolism, which in turn causes damage to proteins, lipids and DNA and activates the DNA damage response. The body can remove damaged mitochondria through mitochondrial autophagy, thus effectively mitigating cellular damage [1]. The function of mitochondria is related to their structure. In general, mitochondria are short rods or spheres, and are small vesicles with a double membrane structure, consisting of an outer membrane, a membrane gap, an inner membrane and a matrix. The outer membrane is generally smooth and continuous and simple in structure, while the inner membrane is highly folded to form cristae, and both membranes allow for specific metabolism, the outer membrane being used for transport of larger proteins and the inner membrane being rich in electron transport chain proteins responsible for ATP synthesis [2].

In general, mitochondria are not static organelles, they change their shape, position and number according to the specificity of the energy required by the living organism and are in dynamic change, maintaining their stability through division and fusion. Mitochondria rely on their own quality control in meeting cellular metabolic demands, such as removing damaged mitochondria through autophagy and replenishing the mitochondrial pool by dividing to produce new mitochondria.

At the same time, mitochondria are relevant to inflammation, vascular health, and the treatment of kidney disease. In this article, we will discuss the association of mitochondria with the above health problems and the countermeasures to solve them in a comprehensive manner.

\section{Cellular Senescence and Related Diseases}

Cellular senescence is the process of decline of cellular functions in the execution of life activities, which is closely associated with the development of various diseases, such as various neurodegenerative diseases, inflammation, tumors, vascular diseases and organ dysfunctions [2]. The most obvious manifestations of aging are the sagging of human skin, memory loss, accumulation of cytoplasmic

a39913954@qq.com 
lipofuscin, graying of hair, and abnormalities in the function of various tissues and organs of the body and the development of diseases [1]. Various factors have been found to influence aging, such as somatic mutations, damage from free radicals, problems with immune function, damage to chromosomal telomeres, effects from the biological clock, and toxicity caused by environmental substances, among other causes.

Aging is caused by a variety of factors, including inflammation and degeneration of body organs and functional decline. In the following, we will discuss the effects of three typical aging-related diseases, namely kidney disease, vascular aging, and inflammation, respectively, and then discuss how to use the relationship between mitochondria and aging to alleviate aging.

\subsection{Nephropathy}

Kidney disease, a common disease, is a serious threat to human health. Mitochondrial integrity is quite important for normal kidney function, and aging happens to be one of the most important pathogenic factors of kidney disease. During the aging process, the structure of the kidney undergoes progressive changes thus making the probability of kidney disease greatly increased. As the main metabolic organ of the body, a large amount of toxic substances and waste are excreted through the urine produced by the kidneys, which improves the body's resistance and immunity. Therefore, the progressive changes in the structure and function of the kidneys in the elderly make them more susceptible to diabetes and chronic kidney disease compared to the young. The kidney is an important and highly energy-consuming organ in the body, and the decline in energy is the basis for the general weakness of the elderly. The presence of kidney disease therefore reduces the flow of energy to other organs, which underlies the development of metabolic and degenerative diseases in other organs [3]. In conclusion, the weakening of the kidneys and aging contribute to each other, ultimately leading to a shorter life span.

\subsection{Vascular aging}

As we age, degenerative changes in the structure of blood vessels occur, eventually leading to disease. And diseases also accelerate the aging of living organisms. Mitochondria will have a small amount of electron leakage during energy supply thus generating ROS, and a small amount of ROS can act as a signaling molecule to help regulate mitochondrial mass. It also activates the antioxidant system and helps cell proliferation, differentiation or apoptosis [4]. However, excessive ROS induce the release of large amounts of ROS from surrounding mitochondria through "reactive oxygen species-induced reactive oxygen species release", leading to the rupture of the outer mitochondrial membrane, forced opening of the mitochondrial permeability transition pore, intracellular calcium overload and DNA damage, which eventually leads to apoptosis or necrosis, thus inducing the development of aging-related diseases. ROS can also directly damage telomeres and inhibit telomerase activity. On the other hand, elevated vascular mitochondrial ROS due to ageing is the main mechanism causing vascular pathological changes [6]. Also, decreased mitochondrial quality due to impaired mitochondrial biosynthesis in aging vascular endothelial cells and smooth muscle cells is another cause of vascular mitochondrial dysfunction.

\subsection{Inflammation}

Inflammation is a defense mechanism resulting from microbial infection, and Matzinger's "danger theory" of inflammation suggests that DAMPs (cell damageassociated molecular patterns) can bind and activate pattern recognition receptors in the cell membrane and cytoplasm, and that when cells are damaged, they can induce a decrease in mitochondrial function through the release of excess DAMPs. cysteinyl aspartate specific proteinase 1 (caspase 1) activation and release of pro-inflammatory cytokines [7] [8]. Chronic inflammation is also a marker of aging. Mitochondrial DNA (mtDNA), as one of the molecular patterns associated with cellular damage, is considered as a marker of the functional link between mitochondrial damage and systemic inflammation by binding to and activating the corresponding receptors, which are involved in various inflammatory responses [9] [10].

The mitochondrial inner membrane complex reduces a large number of oxygen molecules to water during electron transfer, and the remaining $1 \%-2 \%$ of oxygen molecules are reduced to superoxide anions, which once produced in excess can cause oxidative stress and eventually lead to energy failure, inflammation, apoptosis or death [8].

\section{Mitochondria and Aging}

Since mitochondria function as the cellular "energy factory", damage to them can cause dysfunction and result in excessive production of reactive oxygen species (ROS). While mild reactive oxygen species contribute to cell survival, stimulate cellular damage response, proliferation and better metabolism based on toxic excitatory effects, the toxicity caused by excessive ROS will damage cells [11]. The increased production of ROS also affects the stability of mitochondrial lipids and proteins, causing irreversible damage to mitochondrial membranes and dysfunctional mitochondrial oxidation. These damages and dysfunctions would then lead to overexpression of genes in other structures of the body, resulting in over-proliferation of cells[12]. As in vascular smooth muscle cells, this leads to thickening of the cardiovascular lining and eventually to atherosclerotic damage [13]. 


\subsection{Mitochondrial function decreases with age}

The main function of mitochondria is to regulate many different metabolic and signaling pathways and to play an important role in programmed cell death. When considering the impact of mitochondrial dysfunction on aging, it is important to note that mitochondrial biogenesis is controlled at many different levels [14].

\subsubsection{Mitochondrial DNA (mtDNA) mutation accumulation}

Studies have shown that the number of mtDNA mutations increases with age, and in general mutations in mtDNA may be caused by unrepaired DNA damage, such as that caused by ROS [14]. It is therefore hypothesized that de novo mutations in mitochondrial DNA observed during aging are caused by the accumulation of senescent mitochondria and unrepaired damage, but replication errors are now thought to be the more likely cause [15] [16]. This is because most mouse mtDNA mutations arise from replication errors during development, rather than from the accumulation of damage during adulthood [17]. Although it has been known for more than two decades that mtDNA mutations can cause disease and that somatic mtDNA mutations increase with age, experimental data supporting a role for mtDNA mutations in aging were recently obtained in mtDNA mutant mice [17].

\subsubsection{ROS and aging}

The electron respiratory chain consisting of the mitochondrial oxidoreductase complex generates ROS along with ATP, and many studies have shown that the rate of mitochondrial ROS production is negatively correlated with maximum lifespan. Therefore, we can reasonably speculate that mitochondria are associated with senescence. It has been shown that the number of cells decreases during aging, and it is now believed that mitochondria play an important role in the apoptotic process. For example, mitochondria release apoptosis-inducing proteins, and Newmeyer showed through studies on cell-free systems that changes in nuclear morphology during apoptosis can only occur in the presence of mitochondrial homogenates, which contain apoptosis-related proteins, including cytochrome $\mathrm{C}$, apoptosis-inducing factor (AIF), between the inner and outer mitochondrial membranes. Usually, the release of cytochrome $\mathrm{C}$ and AIP requires a change in mitochondrial membrane potential $(\Delta \Psi \mathrm{m})$ and the opening of the mitochondrial permeability transition pore (mPTP) [14] [18] [19].

Even though some scholars such as Ana Bratic, NilsGöran Larsson argue that ROS bring nothing more than a physiological level of oxidative damage that mitochondria can cope with, the free radical theory is probably still the most powerful theory to explain aging in many species [20]. Certainly, there are many short- or long-lived organisms that remain viable primarily through altered mitochondrial metabolism, reactive oxygen species generation, or oxidative stress resistance. This consistency across numerous species does not prove causality, but at least suggests a strong potential relationship. Other observations suggest that a link between ROS levels and the rate of aging can be found by directly stimulating with oxidants that can mimic many cellular and transcriptional level changes. In Harman's original hypothesis, he suggested that aging and aging-related diseases are regulated by intracellular free radicals. Recent findings that certain metabolic genes involved in the TCA cycle can act as tumor suppressors and that genes that slow down overall aging can also slow down the development of chronic diseases such as atherosclerosis suggest that Harman's original idea was correct [21][22] . The above studies have greatly contributed to our understanding of the link between mitochondrial dysfunction and aging, and one can achieve the goal of protecting mitochondria and delaying aging through quality control and protection of mitochondria to delay aging and treat some diseases brought about by mitochondrialmediated aging.

Mitochondrial structural and functional damage has a major impact on nephropathy, vascularity and inflammation and ultimately leads to aging, we discuss below some of the external interventions to achieve mitochondrial protection and repair and thus delay aging.

\subsection{Targeting Mitochondria in Aging Treatment}

Mitochondria are closely related to aging, therefore, using mitochondria and their properties, we can enhance the ability of mitochondria to synthesize ATP through appropriate external interventions, thus enhancing the ability of the body to resist aging; at the same time, reasonably reducing the production of ROS and reducing its damage to cells can also achieve the purpose of using mitochondria to slow down cellular aging and ultimately prolong life span.

\subsubsection{SIRT3}

SIRT3 is a silencing information regulator that reduces ROS production and thus regulates mitochondrial endostasis. It has deacetylating activity towards a range of metabolic targets and is involved in a variety of physiological and disease processes, such as interaction with electron transport complexes I, II and V and regulation of subunits in the electron transport chain and involvement in fatty acid oxidation, amino acid metabolism and redox homeostasis, and interaction with the tricarboxylic acid cycle enzyme isocitrate dehydrogenase 2, which ultimately reduces ROS production [27] [29]. And as a regulatory factor, when the main energy supply substances are simultaneously insufficient, by controlling the amount of SIRT3 can regulate the transcription of respiratory complexes, mitochondria convert glycolysis into fatty acids, amino 
acids and acetate catabolism to maintain the normal production of ATP as an alternative energy source, and this metabolic conversion is the Warburg effect [30]. Regarding the mechanism of action of SIRT3, it can prevent apoptosis by preventing the opening of the mitochondrial permeability transition pore through the deacetylation of procyclin D in cardiac myocytes and reducing the production of ROS, thus avoiding ROS to disturb the calcium homeostasis inside the cell [31]. Thus, SIRT3 maintains normal mitochondrial function, prevents energy failure, and also makes it easier to clean up superoxide. It has been shown that SIRT3 facilitates mitochondrial fusion and cell survival in cardiomyocytes by deacetylating optic atrophy protein 1 [33].Overexpression of SIRT3 improves renal function, regulates oxidative damage, inhibits inflammatory damage, and reduces apoptosis in renal tubular epithelial cells; therefore, it is suggested that restoration of SIRT3 by altering the extracellular signal-regulated kinase-optic atrophy protein 1 cascade, activation of mitochondrial division may be a new approach to treat renal ischemiareperfusion injury [3]. Since AKI (acute kidney injury) has a very high morbidity and mortality rate, this approach may be effective in ensuring kidney health and thus delaying aging.

\subsubsection{Mito $Q$ and $\alpha$-lipoic acid}

The mitochondria-targeted antioxidants MitoQ and $\alpha$-lipoic acid were both found to significantly improve age-related vascular endothelial dysfunction in mice [33][23].MitoQ is a mitochondria-targeted coenzyme, a natural antioxidant that binds to the lipophilic cation decyltriphenylphosphine (TPP), which accumulates in the mitochondrial membrane and blocks mitochondrial oxidative damage [4]. Numerous animal tests have shown that MitoQ has antioxidant effects on a variety of tissues, and results have also shown that it can be made into an effective drug for action in humans.MitoQ can be safely administered for up to one year due to the minimal liver damage caused by controlled doses of MitoQ. Oral administration of MitoQ and related mitochondria-targeted antioxidants also attenuates oxidative damage to mitochondria [34]. Lipoic acid is a mitochondrial coenzyme present in mitochondria that eliminates free radicals responsible for accelerated aging and pathogenesis and catalyzes the production and transfer of acyl groups in the pyruvate dehydrogenase complex and the $\alpha$-ketoglutarate dehydrogenase complex. Because of its high electron density properties, lipoic acid possesses a remarkable ability to react with free radicals and therefore has strong antioxidant properties [23].

\subsubsection{Intermittent fasting}

Intermittent fasting is effective in reducing the production of free radicals, improving glucose regulation, stress response capacity, and suppressing inflammation. During fasting cells can enhance their intrinsic defense against oxidative and metabolic stress and remove or repair damaged parts. The body responds to intermittent fasting by minimizing anabolic processes (synthesis, growth and reproduction), supporting maintenance and repair systems, enhancing stress resistance, reusing damaged molecules, stimulating mitochondrial autopoiesis and promoting cell survival, all of which contribute to improved health and increased disease resistance, and so effectively mitigate damage to mitochondria. Restricting caloric intake stimulates mitochondrial biogenesis and uncoupling, thus mitigating aging. This is because humans have undergone a long evolutionary search for food within an ecological niche where food resources are scarce, allowing most of our organ systems to respond to intermittent fasting in a way that allows the organism to tolerate or overcome challenges and finally restore homeostasis [35]. At the same time, intermittent line fasting enhances neuronal stress resistance, enhances mitochondrial function and stimulates autophagy, antioxidant defense and promotes DNA self-repair through multiple mechanisms [36] [37]. Pu Rongxi et al. showed experimentally that caloric control delayed skeletal muscle aging in mice through the AMPK-SIRT-1 mitochondrial pathway and significantly increased the expression of peroxisome proliferator-activated receptor $\gamma$ coactivator $1 \alpha$, which ultimately promoted mitochondrial production. Meanwhile aerobic exercise alleviates aging by activating the mitochondrial autophagy mechanism [38].

\subsubsection{Biological Molecules}

Natural products have always been an important source of bioactive substances and useful drugs. Among them, phenols, terpenoids, ketol glycosides \& acids are predominant and have an important role in mitochondrial repair and aging mitigation [39]. Phenolic substances have strong antioxidant properties due to their special structure, such as resveratrol, which has some mitochondrial toxicity but delays aging due to toxic excitatory effects that improve mitochondrial respiration, and it can induce mitochondrial autophagy to delay vascular aging. Terpenoids can act on a variety of mitochondria-related genes, for example, oleanolic acid can reduce ROS levels in nematodes and thus prolong their life span. Dehydrofirmanoic acid not only prolongs nematode lifespan and prevents lipofuscin accumulation, but also blocks collagen secretion from human skin fibroblasts. Natural product active molecules of ketol glycosides also have anti-aging activity, such as epimedoside, which can act through at least two mechanisms, either by inducing SOD enzyme activity and reducing malondialdehyde levels to reduce oxidative stress, or by reducing double-stranded DNA breaks and downregulating DNA damage-responsive genes to maintain genomic stability [40]. Both epimedoside and its metabolic derivative, epimedoside hypoglycoside (Icariside II, the in vivo bioactive form of epimedoside), prolong the life span of nematodes. And among acids, chicory acid can act as an antioxidant component in L6 rat myoblasts to scavenge 
ROS and prevent their accumulation and increase mitochondrial biosynthesis and inhibit the insulin/Akt/mTOR signaling pathway [41].

\section{Conclusion and Future Direction}

Ensuring mitochondrial health is effective in treating some of the diseases associated with aging and in mitigating aging because of their highly specialized structure and their involvement in energy synthesis. Mitochondrial health is associated with the oxygen radical theory of aging, and the mitochondria themselves maintain proper function by stimulating autophagy. Inevitably, some diseases arise during the body's decline, and these diseases also accelerate aging, such as kidney disease, which damages the kidneys, the main energy-consuming organ, and accelerates aging; vascular aging, which leads to impaired material transport and mitochondrial dysfunction; and inflammation, which is a hallmark of aging, caused by excess superoxide anions and also by apoptosis. The use of SIRT3 can improve renal function, reduce ROS production, regulate oxidative damage and inhibit inflammation; MitoQ and $\alpha$-lipoic acid can also effectively block oxidative damage in mitochondria due to their strong antioxidant properties. Intermittent fasting can improve the body's stress capacity, while reducing free radical production and inhibiting inflammation. Some other bioactive macromolecules also have antioxidant properties and increase mitochondrial biosynthesis. Although some studies suggest that the free radical theory is not the ultimate cause of aging, the accumulation of oxidative damage to mitochondria with aging is currently the relatively widely accepted theory, while it has also been suggested that mtDNA replication errors cause mitochondrial damage. With such agents, it seems that a better way to alleviate aging is by treating some of the aging diseases mediated by mitochondria.

\section{References}

1. Cheng J, Liu G. The relationship between mitochondria and aging and countermeasures against aging [J]. Journal of Practical Diabetes, 2019, 15(01):6-8.

2. Wang $\mathrm{Y}$, et al. The role of mitochondria in cellular senescence[J]. Medical Review, 2019, 25(08):14571462.

3. Chen L, Yang D. Advances in the study of mitochondrial SIRT3 in kidney disease[J]. Medical Review, 2020, 26(13):2502-2506.

4. Chen Y, et al. Mitochondrial quality control and vascular aging[J]. Advances in Cardiovascular Diseases, 2019, 40(04):586-590.

5. Oelze M, Kroller-Schon S, Steven S, et al.Glutathione peroxidase-1 deficiency potentiates dysregulatory modifications of endothelial nitric oxide synthase and vascular dysfunction in aging[J].Hypertension, 2014, 63: 390-396.

6. Ungvari Z, William ES, Anna C. Mitochondria and aging in the vascular system [J]. J Mol Med (Berl), 2010, 88( 10):1021-1027.

7. Matzinger P. Tolerance, danger, and the extended family[J]. Annu Rev Immunol, 1994, 12( 1) : $991-$ 1045. DOI: 10.1146 /

8. Liu J, Zhang L. Mitochondrial dysfunction and the correlation between inflammation and aging[J]. Chinese Journal of Geriatric Multiorgan Diseases, 2019, 18(06):469-472.

9. Caielli S, Athale S, Domic B, et al. Oxidized mitochondrial nucleoids released by neutrophils drive type I interferon production in human lupus [ J]. J Exp Med, 2016, 13( 5) : 697 - 713. DOI: 10.1084/jem.20151876.

10. Wu J, Sun L, Chen X, et al. Cyclic GMP-AMP is an endogenous second messenger in innate immune signaling by cytosolic DNA [ J]. Science, 2013, $339(6121)$ : $826-830$. DOI: 10.1126/ science.1229963.

11. Cichoz-Lach H, Michalak A. Oxidative stress as a crucial factor in liver diseases[J]. World J Gastroenterol, 2014, 20 (25) :8082-8091.

12. Linnane AW, Marzuki S, Ozawa T, et al. Mitochondrial DNA mutations as an important contributor to ageing and degenerative diseases[J]. Lancet, 1989, 1(8639): 642-645

13. Lee J Y, Jung G Y, Heo H J, el al.4-hydroxynonenal induces vascular smooth muscle cell apoptosis through mitochondrial generation of reactive oxygen species[J]. Toxical Lett, 2006, 166:212

14. Bratic A, Larsson NG. The role of mitochondria in aging. J Clin Invest. 2013;123(3):951-957. doi:10.1172/JCI64125

15. Stewart JB, et al. Strong purifying selection in transmission of mammalian mitochondrial DNA. PloS Biol. 2008;6(1): e10

16. Stewart JB, Freyer C, Elson JL, Larsson N-G. Purifying selection of mtDNA and its implications for understanding evolution and mitochondrial disease. Nat Rev Genet. 2008;9(9):657-662.

17. Ameur A, et al. Ultra-deep sequencing of mouse mitochondrial DNA: mutational patterns and their origins. PLoS Genet. 2011;7(3): e1002028.

18. Votyakova TV, R eynolds L J. Deltapai(m) Depandentad independent prodation of reactive oxygen species by ratbrainmito chondria. Neuro chem 2001; 79(2): $266-277$.

19. Barja G. Aging invertebrates and the effect of coloricre atriction: amitochondrial free radical production 
mtDNA damage mechanism. Biol Rev 2004; 79: 235 $-251$.

20. Napoleone P, Ferrante F, Chirardi O. Age-dependent nerve cell loss in the brain of spragre-Dawley rats: Effect of long terms acetyl L-carnitine treatment. Arch Gerontol Geriatr 1999; 10: 173 - 185.

21. Pollard, P.J., Wortham, N.C., and Tomlinson, I.P. (2003). The TCA cycle and tumorigenesis: the examples of fumarate hydratase and succinate dehydrogenase. Ann. Med. 35, 632-639.

22. Napoli, C., Martin-Padura, I., de Nigris, F, Giorgio, M., Mansueto, G., Somma, P, Condorelli, M., Sica, G., De Rosa, G., and Pelicci, P.(2003). Deletion of the p66Shc longevity gene reduces systemic and tissue oxidative stress, vascular cell apoptosis, and early atherogenesis in mice fed a high-fat diet. Proc. Natl. Acad. Sci. USA100, 2112-2116.

23. Ying $Z$, Xie $X$, Chen $M$, et al.Alpha-lipoic acid activates eNOS through activation of PI3-kinase /Akt signaling pathway[J].Vascul Pharmacol, 2015, 64: 2835.

24. Newmeyer DD, Farschon DM, R eed TC. Cell-free opoptosis in xenopus egg extracts: lnhibition by bel-2 and requirement for an organelle fraction neriched in mitochondria Cell 1994; 24: 353 - 364

25. Robert S. Balaban, Shino Nemoto, Toren Finkel. Mitochondria, Oxidants, and Aging[J]. Cell, 2005, 120(4).

26. Rahman M, Nirala NK, Singh A, et al. Drosophila Sirt2 /mammalian SI R T3 deacetylates ATP synthaseßand regulates complex V activity[J]. J Cell Biol, 2014, 206(2): 289-305.

27. Ahn BH, Kim HS, Song S, et al. A role for the mitochondrial deacetylase Sirt3 in regulating energy homeostasis[J]. Proc Natl Acad Sci U S A, 2008, 105(38): 14447-14452.

28. Finley LW, Haas W, Desquiret-Dumas V, et al. Succinate dehydrogenase is a direct target of sirtuin 3 deacetylase activity[J]. PLoS One, 2011, 6(8): e23295.

29. Kumar S, Lombard DB. Mitochondrial sirtuins and their relationships with metabolic disease and cancer[J]. Antioxid Redox Signal, 2015, 22( 12) : 1060-1077.

30. Guarente L. The many faces of sirtuins: Sirtuins and the Warburg effect[J]. Nat Med, 2014, 20(1) : 24-25.

31. Bochaton T, Crola-Da-Silva C, Pillot B, et al. Inhibition of myocardial reperfusion injury by ischemic postconditioning requires sirtuin 3-mediated deacetylation of cyclophilin D[J]. J Mol Cell Cardiol, 2015, 84: 61-69.

32. Samant SA, Zhang HJ, Hong $Z$, et al. SI R T3 deacetylates and activates OPA1 to regulate mitochondrial dynamics during stress[J]. Mol Cell Biol, 2014, 34(5) : 807-819.
33. Gioscia- R yan R A, Larocca TJ, Sindler AL, et al. Mitochondria-targeted antioxidant (MitoQ) ameliorates age-related arterial endothelial dysfunction in mice $[\mathrm{J}]$. J Physiol, 2014, 592( 12) : 2549-2561.

34. Robin A.J. Smith, Michael P. Murphy. Animal and human studies with the mitochondria-targeted antioxidant MitoQ[J]. Annals of the New York Academy of Sciences, 2010, 1201(1).

35. Rafael de Cabo, Ph.D., and Mark P. Mattson, etc.Effects of Intermittent Fasting on Health, Aging, and Disease[J].N Engl J Med, 2019, 381:2541-2551.

36. Mattson MP, Arumugam TV. Hallmarks of brain aging adaptive and pathological modification by metabolic states. Cell Metab 2018; 27:1176-1199.

37. Menzies FM, Fleming A, Caricasole A, et al. Autophagy and neurodegeneration: pathogenic mechanisms and therapeutic opportunities. Neuron 2017; 93:1015-1034.

38. $\mathrm{Pu} \mathrm{R}$, et al. Caloric restriction delays skeletal muscle aging in mice via AMPK-SIRT1-mitochondrial pathway. Journal of the Third Military Medical University, 2017, 39(6): 548-552.

39. Tong Q, Wang Z, Huang X. Mitochondrial functionrelated active molecules of anti-aging natural products[J]. Natural Products Research and Development, 2018, 30(09):1649-1654.

40. Zhang SQ, et al. Icariin, a natural flavonol glycoside, extends healthspan in mice[J]. Exp Gerontol, 2015, 69:226235.

41. Schlernitzauer A, et al. Chicoric acid is an antioxidant molecule that stimulates AMP kinase pathway in L6 myotubes and extends lifespan in Caenorhabditis elegans[J]. PLoS One, 2013, 8: e78788. 